\title{
On the Performance of MIMO Nullforming with Random Vector Quantization Limited Feedback
}

\author{
D. Richard Brown III, Senior Member, IEEE, and David J. Love, Senior Member, IEEE
}

\begin{abstract}
This paper analyzes the performance of random vector quantization (RVQ) for limited feedback nullforming in multi-input multi-output (MIMO) communication systems with and without receiver coordination. A single-stream scenario is considered in which one or more primary receivers request nulls by providing limited feedback to the transmitter. Without receiver coordination, each primary receiver informs the transmitter of its best beamforming precoding vector. The transmitter then selects a zero-forcing precoding vector orthogonal to all of the beamforming precoding vectors. With receiver coordination, the primary receivers feed back the common precoding vector that minimizes the average interference. In both cases, secondary receivers in the network do not provide feedback and experience channels statistically equivalent to a single-antenna fading channel. Analytical results show that, for a system with $K$ primary receivers and random codebooks with $N=2^{B}$ precoding vectors, the mean received power at the primary receivers is upper bounded by $N^{-1 / K}=2^{-B / K}$ with or without receiver coordination. Exact results are also derived for the $K=1$ receiver case. Numerical results verify the scaling and also show that systems with receiver coordination outperform those without receiver coordination by a constant gap for large $N$ in terms of average interference.
\end{abstract}

Index Terms-Antenna arrays, nullforming, zero-forcing, limited feedback, random vector quantization, MIMO communication, interference mitigation.

\section{INTRODUCTION}

$\mathbf{I}$ $\mathrm{N}$ wireless communication systems, antenna arrays can be used to steer transmitted signals toward intended receivers and away from unintended receivers. The benefits of antenna arrays and directional transmission are manifold and include increased range, increased rate, increased security, and reduced interference [1]-[4]. The potential for steering nulls [5] to avoid interference in certain directions is particularly appealing because, unlike strong beams which require many antenna elements, deep nulls can be achieved with as few as two antenna elements. In other words, nullforming can provide significant margin between the power received at intended and unintended receivers with small arrays.

Both beamforming and nullforming require channel state knowledge at the transmitter to achieve the desired directivity

Manuscript received June 11, 2013; revised October 4 and December 18, 2013; accepted February 3, 2014. The associate editor coordinating the review of this paper and approving it for publication was X. Zhou.

This work was supported by the National Science Foundation awards CCF1302104 and CCF-1319458.

D. R. Brown III is with the Department of Electrical and Computer Engineering, Worcester Polytechnic Institute, Worcester MA 01609 USA (email: drb@wpi.edu).

D. J. Love is with the School of Electrical and Computer Engineering, Purdue University, West Lafayette IN 47907 USA (e-mail: djlove@ecn.purdue.edu).

Digital Object Identifier 10.1109/TWC.2014.040914.131052 pattern. In some cases, e.g., time division duplexed (TDD) channels with reciprocal transceivers, channel estimates can be inferred from channel reciprocity. More often, however, it is necessary to estimate the channels at the receiver and provide some sort of feedback to the transmitter to facilitate multi-antenna transmission with a desired directivity pattern [6]. Since feedback creates overhead, several limited-rate feedback techniques have been developed recently for multi-input multi-output (MIMO) and multi-input single-output (MISO) systems, for example see [7]-[20] and the references therein. These techniques are typically based on vector quantization and usually require carefully designed precoding vector codebooks to achieve good performance with low feedback overhead.

Among the various limited-rate feedback techniques, random vector quantization (RVQ) is appealing since the codebook, which is known to both the transmitter and the receiver, is randomly generated independently of the channel realization. RVQ was first proposed in [21], [22] and was shown to be asymptotically optimal for beamforming as $M, N \rightarrow \infty$ in [23], [24] where $M$ is the number of transmit antennas and $N$ is the number of elements in the precoding vector codebook. RVQ has received further analysis in [25]-[29]. The performance of RVQ for a MISO beamforming system was studied in [30] where it was shown that the beamforming loss due to RVQ was on the order of $N^{-\frac{1}{M-1}}$. Note that, in beamforming systems with a total transmit power constraint, the received power grows linearly with $M$. Hence, to achieve a significant margin between the power received at intended and unintended receivers in a beamforming system, $M$ must be large. The results in [30] imply, however, that when $M$ is large the codebook size $N$ must also be large to avoid significant beamforming loss from RVQ with respect to unquantized channel estimates.

In this paper, we analyze the performance of MIMO nullforming with RVQ limited feedback in systems with and without receiver coordination. Our focus is on a single-stream scenario where one or more "primary" receivers provide feedback to the transmitter to facilitate nullforming toward these receivers. "Secondary" receivers in the network do not provide feedback to the transmitter and experience channels statistically equivalent to a single-antenna fading channel. Examples of applications under this model include (i) jamming, (ii) cognitive radio, and (iii) overlay networks. In the jamming scenario, the primary receivers are "friendly" receivers that provide feedback to avoid being jammed and the secondary receivers are "unfriendly" receivers that we wish to jam. In the cognitive radio and overlay network scenarios, the transmitter 
in the secondary network receives feedback from users in the primary network (the primary receivers) and steers nulls toward these users while transmitting a single stream to users in the secondary network (the secondary receivers). Since the statistics of the received power at the secondary users are independent of the transmitter's normalized precoding vector, our analysis focuses on quantifying the mean received null power at the primary receivers as a function of the number of transmit antennas $M$, number of primary receivers $K<M$, and RVQ codebook size $N$.

For systems without receiver coordination, we analyze an approach similar to [17] where each primary receiver feeds back the index of its best beamforming vector and the transmitter computes a zero-forcing precoding vector orthogonal to all of the beamforming vectors. For codebooks with $N=2^{B}$ codewords, this approach requires a total of $K B$ bits of feedback. Note that [17] considers a multi-stream scenario with $K=M$ and characterizes the SINR at each receiver. In this paper, we consider a single-stream scenario with $K<M$ primary receivers where the goal is to minimize the mean received power at the primary receivers.

For systems with receiver coordination, the primary receivers exchange channel estimates and select the precoding vector from a common codebook that minimizes the average interference across all of the primary receivers. This approach requires less feedback ( $B$ bits rather than $K B$ bits) and also directly optimizes the performance metric through the feedback of the best joint nullforming vector, rather than indirectly computing a precoding vector orthogonal to the individual beamforming vectors.

Our main contribution is the development of scaling results for the mean received power, i.e., the average interference, at the primary receivers. In particular, we show that the mean received power at the primary receivers has an upper bound that scales as $N^{-1 / K}=2^{-B / K}$ with or without receiver coordination. In other words, a $3 \mathrm{~dB}$ reduction in mean received power can be achieved by increasing the number of RVQ codebook vectors by a factor of $2^{K}$. While the analysis leads to identical bounds on the mean received power for nullforming with and without receiver coordination, we also derive exact results for the $K=1$ receiver case that are different for systems with and without receiver coordination and provide numerical results showing that systems with receiver coordination outperform those without receiver coordination by a constant gap for large $N$ in terms of mean received power. This gap can be overcome by using larger codebooks in systems without receiver coordination.

The paper is organized as follows. Section II provides an overview of the nullforming systems under consideration, including with and without receiver coordination. A probabilistic analysis of the average null depth is presented in Section III. Simulation results verifying the analysis are provided in Section IV. We conclude in Section V.

Notation: Vectors and matrices are denoted by boldface letters. $\mathbb{R}, \mathbb{C}$, and $\mathbb{N}+$ denote the real, complex, and positive natural numbers, respectively. The matrix $\boldsymbol{I}$ and the vector $\boldsymbol{e}_{k}$ denote identity matrix and the $k^{\text {th }}$ standard basis vector corresponding to the $k^{\text {th }}$ column of $\boldsymbol{I}$. $\|\cdot\|$ represents the Euclidean norm of the enclosed vector. We use $\mathrm{E}[\cdot]$ and

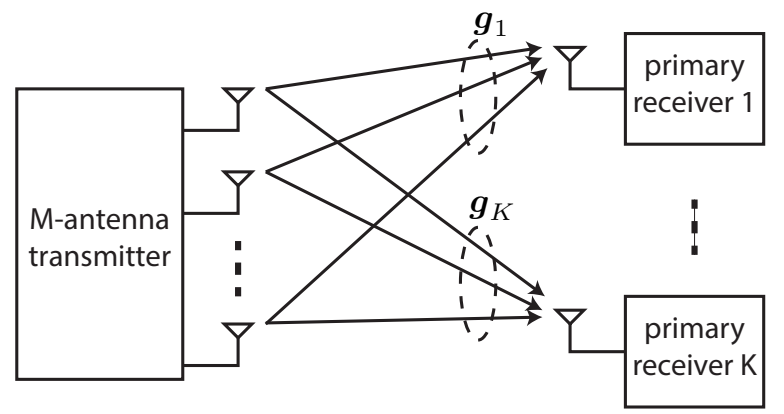

Fig. 1. MIMO system model. Secondary receivers are not shown.

$(\cdot)^{H}$ for expectation and complex conjugate transposition, respectively. We also use

$$
\Gamma(x)=\int_{0}^{\infty} t^{x-1} e^{-t} d t .
$$

to denote the Gamma function and

$$
\beta(s, t)=\frac{\Gamma(s) \Gamma(t)}{\Gamma(s+t)}
$$

to denote the beta function [31].

\section{SYSTEM MODEL}

We consider the MIMO system shown in Fig. 1 with an $M \geq 2$ antenna transmitter and $1 \leq K<M$ single-antenna primary receivers.

The vector channel from the transmitter to primary receiver $k$ is denoted as $\boldsymbol{g}_{k} \in \mathbb{C}^{M \times 1}$. It is assumed that $\mathrm{E}\left[\boldsymbol{g}_{k}\right]=0$ and

$$
\mathrm{E}\left[\boldsymbol{g}_{j} \boldsymbol{g}_{k}^{H}\right]= \begin{cases}2 \boldsymbol{I} & j=k \\ 0 & \text { otherwise }\end{cases}
$$

where each element of $\boldsymbol{g}_{k}$ is a proper complex Gaussian random variable with independent unit-variance real and imaginary parts. We also define the normalized channel from the transmitter to primary receiver $k$ as

$$
\tilde{\boldsymbol{g}}_{k}=\frac{\boldsymbol{g}_{k}}{\left\|\boldsymbol{g}_{k}\right\|}
$$

and the normalized MIMO channel matrix

$$
\tilde{\boldsymbol{G}}=\left[\tilde{\boldsymbol{g}}_{1}, \ldots, \tilde{\boldsymbol{g}}_{K}\right] \in \mathbb{C}^{M \times K} .
$$

The primary receivers are assumed to have error-free feedback links to the transmitter for the purpose of conveying one or more precoding vector indices. These precoding vector indices are then used by the transmitter to compute/select a precoding vector $\boldsymbol{w} \in \mathbb{C}^{M \times 1}$ such that the interference is minimized at the primary receivers. Using this set-up, we assume that the $k$ th user has input-output equation

$$
y_{k}=s \boldsymbol{w}^{H} \boldsymbol{g}_{k}+z_{k}
$$

where $y_{k}$ is the received signal, $s$ is the transmitted signal with $E\left[|s|^{2}\right]=P$, and $z_{k}$ is additive white Gaussian noise distributed as $\mathcal{C N}\left(0, \sigma^{2}\right)$.

We can further denote the channel to a secondary receiver as $\boldsymbol{g}_{0}$. We assume $\boldsymbol{g}_{0}$ is independent and identically distributed with respect to the primary receiver channels $\left\{\boldsymbol{g}_{k}\right\}$ and that 
the secondary receiver does not provide feedback to the transmitter. Since the transmitter's precoding vector $\boldsymbol{w}$ is computed only as a function of the primary receiver channels $\left\{\boldsymbol{g}_{1}, \ldots, \boldsymbol{g}_{K}\right\}$, it is independent of $\boldsymbol{g}_{0}$. This implies that the effective channel at the secondary receiver is equivalent in distribution to a single antenna Rayleigh fading channel with mean received power $\mathrm{E}\left[\left|\boldsymbol{g}_{0}^{H} \boldsymbol{w}\right|^{2}\right]=2$ assuming unit-norm precoding vectors.

Since the received power distribution at a secondary receiver is independent of the precoding vector, our focus is on analyzing the effect of feedback on the mean received power at the primary receivers. The following sections describe the feedback protocols with and without receiver coordination.

\section{A. Nullforming without Primary Receiver Coordination}

In the absence of receiver coordination, a zero-forcing scheme similar to [17] can be used to steer nulls toward the primary receivers. Each primary receiver $k$ feeds back $B$ bits that represent a quantized version of the channel's direction $\tilde{\boldsymbol{g}}_{k}$. The transmitter then computes a precoding vector that steers nulls toward all $K$ reconstructed channels.

Note that $\tilde{\boldsymbol{G}}$ has at most $\operatorname{rank} K$. This means that we can always find an $M \times(K+1)$ orthonormal matrix $\boldsymbol{A}_{\text {opt }}$ such that each $\boldsymbol{g}_{k}$ lies in the column space of $\boldsymbol{A}_{\text {opt }}$. If the transmitter and primary receivers somehow had access to $\boldsymbol{A}_{o p t}$, each primary receiver $k$ could project $\boldsymbol{g}_{k}$ onto $\boldsymbol{A}_{o p t}$ and quantize the unit vector $\boldsymbol{A}_{o p t}^{H} \boldsymbol{g}_{k} /\left\|\boldsymbol{A}_{o p t}^{H} \boldsymbol{g}_{k}\right\|$. The transmitter would then be left with the problem of finding the null space of the $(K+1) \times K$ matrix $\boldsymbol{A}_{\text {opt }}^{H} \tilde{\boldsymbol{G}}$. Because the unit sphere in $\mathbb{C}^{K+1}$ when taking into account phase invariance (i.e., the Grassmann manifold of one-dimensional subspaces) is of dimension $K$, rather than $M-1$, each primary receiver's quantizer uses $B / K$ bits per dimension instead of $B /(M-1)$ bits per dimension. This improved bit allocation reduces the channel distortion at the transmit side after reception of the feedback signals.

Unfortunately, the matrix $\boldsymbol{A}_{o p t}$ is not known to the transmitter or the receivers performing quantization. Nevertheless, we can still achieve a $B / K$ bit allocation per dimension by projecting the quantization problem to an arbitrary reduced subspace. We assume each primary receiver has an independently generated codebook of unit-norm randomly generated complex vectors $\mathcal{V}_{k}=\left\{\boldsymbol{v}_{k, 1}, \ldots, \boldsymbol{v}_{k, N}\right\}$ with $\boldsymbol{v}_{k, i}=\boldsymbol{u}_{k, i} /\left\|\boldsymbol{u}_{k, i}\right\|$ and with $\left\{\boldsymbol{u}_{k, 1}, \ldots, \boldsymbol{u}_{k, N}\right\}$ all $(K+1)$-dimensional vectors consisting of independent and identically distributed zeromean, unit-variance complex Gaussian random variables. The transmitter is assumed to know all of the codebooks.

After estimating its vector channel from the transmitter, each primary receiver first computes

$$
\boldsymbol{h}=\boldsymbol{A}^{H} \boldsymbol{g}_{k}
$$

where $\boldsymbol{A} \in \mathbb{C}^{M \times(K+1)}$ is an arbitrary but common unitary matrix satisfying $\boldsymbol{A}^{H} \boldsymbol{A}=\boldsymbol{I}$ that projects the vector channels into a $(K+1)$-dimensional subspace. Each primary receiver then finds the index of the codebook vector that maximizes the inner product with the receiver's reduced-subspace channel, i.e.,

$$
i_{\mathrm{opt}}^{(k)}=\underset{i \in\{1, \ldots, N\}}{\operatorname{argmax}}\left|\boldsymbol{v}_{k, i}^{H} \tilde{\boldsymbol{h}}_{k}\right|^{2}
$$

where

$$
\tilde{\boldsymbol{h}}_{k}=\frac{\boldsymbol{h}_{k}}{\left\|\boldsymbol{h}_{k}\right\|}=\frac{\boldsymbol{A}^{H} \boldsymbol{g}_{k}}{\left\|\boldsymbol{h}_{k}\right\|}=\frac{\left\|\boldsymbol{g}_{k}\right\|}{\left\|\boldsymbol{h}_{k}\right\|} \boldsymbol{A}^{H} \tilde{\boldsymbol{g}}_{k} .
$$

Note that, since $\tilde{\boldsymbol{g}}_{k}$ is isotropic and $\boldsymbol{A}$ is unitary, $\tilde{\boldsymbol{h}}_{k}$ is also isotropic. Each primary receiver then feeds the index $i_{\text {opt }}^{(k)}$ back to the transmitter to facilitate computation of a zero-forcing precoding vector. Note that each primary receiver feeds back $B=\log _{2}(N)$ bits to the transmitter for a total of $K B$ bits of feedback.

After receiving feedback from all of the primary receivers, the transmitter forms the matrix

$$
\boldsymbol{V}=\left[\begin{array}{lll}
\boldsymbol{v}_{1, i_{\mathrm{opt}}^{(1)}} & \cdots & \boldsymbol{v}_{K, i_{\mathrm{opt}}^{(K)}}
\end{array}\right] \in \mathbb{C}^{(K+1) \times K}
$$

from the known RVQ codebooks. Note that the rank of $\boldsymbol{V}$ is at most $K$, hence $\boldsymbol{V}^{H}$ has a nonempty nullspace. The transmitter then computes a unit-norm $M \times 1$ complex precoding vector as $\boldsymbol{w}_{\mathrm{opt}}=\boldsymbol{A} \boldsymbol{v}_{\mathrm{opt}}$ where $\boldsymbol{v}_{\mathrm{opt}} \in \mathbb{C}^{K+1}$ is a unit-norm vector in the nullspace of $\boldsymbol{V}^{H}$. The vector $\boldsymbol{v}_{\text {opt }}$ can be computed, for example, by performing a singular value decomposition on $\boldsymbol{V}^{H}$. The right-singular vectors of $\boldsymbol{V}^{H}$ corresponding to the one or more singular values of $\boldsymbol{V}^{H}$ equal to zero provide an orthonormal basis for the nullspace of $\boldsymbol{V}^{H}$ [32]. Also note that $\left\|\boldsymbol{w}_{\text {opt }}\right\|=1$ since $\boldsymbol{A}^{H} \boldsymbol{A}=\boldsymbol{I}$.

\section{B. Nullforming with Primary Receiver Coordination}

In the case when the primary receivers can coordinate by exchanging channel estimates prior to sending feedback to the transmitter, we assume the transmitter and primary receivers all share a single common codebook of unitnorm randomly generated complex precoding vectors $\mathcal{W}=$ $\left\{\boldsymbol{w}_{1}, \ldots, \boldsymbol{w}_{N}\right\}$ with $\boldsymbol{w}_{i}=\boldsymbol{u}_{i} /\left\|\boldsymbol{u}_{i}\right\|$ and with $\left\{\boldsymbol{u}_{1}, \ldots, \boldsymbol{u}_{N}\right\}$ all $M$-dimensional vectors consisting of independent and identically distributed zero-mean, unit-variance complex Gaussian random variables. Note that the statistics of the codebook are the same as in the case without receiver coordination, but here each codeword is an element of $\mathbb{C}^{M}$ and all of the primary receivers share a common codebook.

After estimating the vector channels from the transmitter, the primary receivers coordinate by exchanging unquantized channel estimates. One or more primary receivers then search through the common codebook to determine the index of the precoding vector that minimizes the average power over all of the primary receivers, i.e.,

$$
i_{\text {opt }}=\underset{i \in\{1, \ldots, N\}}{\operatorname{argmin}} \frac{1}{K} \sum_{k=1}^{K}\left|\boldsymbol{w}_{i}^{H} \tilde{\boldsymbol{g}}_{k}\right|^{2} .
$$

Once the optimal precoding vector is found, only one primary receiver then needs to feed back the integer value of $i_{\text {opt }}$ to facilitate nullforming by the transmitter. In this case, only $B=$ $\log _{2}(N)$ bits of feedback are required. The selected precoding vector is then used directly by the transmitter.

In this context, receiver coordination effectively causes the separate single-antenna primary receivers to behave as a single multi-antenna primary receiver since we assume no loss of information in the exchange of channel estimates. This is equivalent to a $M \times K$ single-user MIMO system for which we wish to minimize the average power received over the $K$ receive antennas. 


\section{Remarks}

We briefly mention here some qualitative differences in nullforming with and without receiver coordination. From the previous discussion, it is evident that receiver coordination can provide (a) reduced feedback overhead ( $B$ total bits versus $K B$ total bits without receiver coordination), (b) reduced requirements for codebook memory at the transmitter due to the use of a common codebook, and (c) reduced computational requirements at the transmitter since the feedback directly specifies the best precoding vector and does not require the transmitter to perform any additional calculations. In particular, with respect to point (b), a system with receiver coordination requires only one codebook irrespective of the number of primary receivers $K$. Without receiver coordination, a family of independent RVQ codebooks must be stored at each primary receiver and at the transmitter for each $K \in\{1, \ldots, M-1\}$.

The tradeoffs of receiver coordination, beyond the obvious requirement for a backhaul or local area network over which channel estimates can be exchanged, include (a) additional computational burden at the primary receivers and (b) potential for increased latency since channel estimates must be exchanged before the optimal precoding vector can be determined. Effectively, receiver coordination trades off feedback overhead, codebook storage space, and transmitterside processing for local area network overhead and receiverside processing. This tradeoff may be appealing in scenarios where feedback overhead is costly.

Finally, note that, with or without receiver coordination, the primary receivers must know the number of transmit antennas $M$ to select the best RVQ precoding vector(s). In the case without receiver coordination, the primary receivers must also know $K$ in order to select the common unitary projection matrix $\boldsymbol{A}$. A table of $\boldsymbol{A}$ matrices, indexed by $M$ and $K$, could be computed and distributed in advance among the primary receivers to facilitate efficient precoding vector calculations in changing network conditions.

\section{ANALYSIS}

This section analyzes the performance of nullforming in terms of the mean normalized received power observed by the primary receivers with and without receiver coordination as a function of the parameters $K, M$, and $N$.

\section{A. Nullforming without Primary Receiver Coordination}

As discussed in Section II-A, in the absence of receiver coordination each primary receiver finds the codebook vector that maximizes the inner product with the receiver's reducedsubspace channel. Denoting the normalized received power at primary receiver $k$ when the transmitter uses the precoding vector $\boldsymbol{w}_{\text {opt }}$ as

$$
\nu_{\mathrm{opt}}^{(k)}=\left|\boldsymbol{w}_{\mathrm{opt}}^{H} \tilde{\boldsymbol{g}}_{k}\right|^{2}
$$

and the average normalized received power as

$$
\nu_{\mathrm{opt}}=\frac{1}{K} \sum_{k=1}^{K} \nu_{\mathrm{opt}}^{(k)}
$$

the following Theorem establishes an upper bound on the expected value of $\nu_{\mathrm{opt}}$.

Theorem 1. In a MIMO system with $K \geq 1$ primary receivers with independently generated $N$-vector $R V Q$ codebooks and $M>K$ transmit antennas, the expected value of the average normalized received power without receiver coordination is upper bounded as

$$
\mathrm{E}\left[\nu_{\mathrm{opt}}\right] \leq \frac{K+1}{M} N \beta\left(N, \frac{K+1}{K}\right) \leq N^{-1 / K}
$$

where $\beta(s, t)$ is the beta function defined in (2).

Proof: From (6), the normalized power at the $k^{\text {th }}$ primary receiver when the precoding vector $\boldsymbol{w}_{\mathrm{opt}}=\boldsymbol{A} \boldsymbol{v}_{\mathrm{opt}}$ is used can be written as

$$
\begin{aligned}
\nu_{\mathrm{opt}}^{(k)} & =\left|\boldsymbol{v}_{\mathrm{opt}}^{H} \boldsymbol{A}^{H} \tilde{\boldsymbol{g}}_{k}\right|^{2} \\
& =\frac{\left\|\boldsymbol{h}_{k}\right\|^{2}}{\left\|\boldsymbol{g}_{k}\right\|^{2}} \cdot\left|\boldsymbol{v}_{\mathrm{opt}}^{H} \tilde{\boldsymbol{h}}_{k}\right|^{2}
\end{aligned}
$$

where the second equality is from (5). Using an orthogonal decomposition [32], we can write

$$
\tilde{\boldsymbol{h}}_{k}=a \boldsymbol{v}_{k, i_{\mathrm{opt}}^{(k)}}+b \boldsymbol{n}_{k}
$$

where $\boldsymbol{n}_{k}$ is a unit vector orthogonal to $\boldsymbol{v}_{k, i_{\text {opt }}^{(k)}}$ and where $a$ and $b$ are scalars denoting the components of $\tilde{\boldsymbol{h}}_{k}$ parallel and orthogonal to $\boldsymbol{v}_{k, i_{\mathrm{opt}}^{(k)}}$, respectively. Denoting the maximum inner product at primary receiver $k$ as

$$
\nu_{\max }^{(k)}=\left|\boldsymbol{v}_{k, i_{\mathrm{opt}}^{(k)}}^{H} \tilde{\boldsymbol{h}}_{k}\right|^{2},
$$

we have

$$
\begin{aligned}
a & =e^{j \theta} \sqrt{\nu_{\max }^{(k)}} \text { and } \\
b & =\sqrt{1-\nu_{\max }^{(k)}}
\end{aligned}
$$

where $\theta$ is the phase of the projection of $\tilde{\boldsymbol{h}}_{k}$ onto $\boldsymbol{v}_{k, i_{\mathrm{opt}}^{(k)}}$. From (9), we can then write

$$
\begin{aligned}
\nu_{\mathrm{opt}}^{(k)} & =\frac{\left\|\boldsymbol{h}_{k}\right\|^{2}}{\left\|\boldsymbol{g}_{k}\right\|^{2}}\left|\boldsymbol{v}_{\mathrm{opt}}^{H}\left(a \boldsymbol{v}_{k, i_{\mathrm{opt}}^{(k)}}+b \boldsymbol{n}_{k}\right)\right|^{2} \\
& =\frac{\left\|\boldsymbol{h}_{k}\right\|^{2}}{\left\|\boldsymbol{g}_{k}\right\|^{2}}\left(1-\nu_{\text {max }}^{(k)}\right)\left|\boldsymbol{v}_{\mathrm{opt}}^{H} \boldsymbol{n}_{k}\right|^{2} \\
& \leq \frac{\left\|\boldsymbol{h}_{k}\right\|^{2}}{\left\|\boldsymbol{g}_{k}\right\|^{2}}\left(1-\nu_{\text {max }}^{(k)}\right)
\end{aligned}
$$

where the second equality results from the fact that $\boldsymbol{v}_{\mathrm{opt}}$ is orthogonal to $\boldsymbol{v}_{k, i_{\text {opt }}^{(k)}}$ for all $k$ and the final inequality results from the fact that both $\boldsymbol{v}_{\text {opt }}$ and $\boldsymbol{n}_{k}$ are unit vectors.

To compute the expected value of this upper bound, we can perform an iterated expectation by first conditioning on $\boldsymbol{g}_{k}$. We have

$$
\begin{aligned}
\mathrm{E}\left[\nu_{\mathrm{opt}}^{(k)} \mid \boldsymbol{g}_{k}\right] & \leq \frac{\left\|\boldsymbol{h}_{k}\right\|^{2}}{\left\|\boldsymbol{g}_{k}\right\|^{2}}\left(1-\mathrm{E}\left[\nu_{\max }^{(k)} \mid \boldsymbol{g}_{k}\right]\right) \\
& =\frac{\left\|\boldsymbol{h}_{k}\right\|^{2}}{\left\|\boldsymbol{g}_{k}\right\|^{2}} N \beta\left(N, \frac{K+1}{K}\right)
\end{aligned}
$$

where we have used the result that

$$
\mathrm{E}\left[\nu_{\max }^{(k)} \mid \boldsymbol{g}_{k}\right]=\mathrm{E}\left[\nu_{\max }^{(k)}\right]=1-N \beta\left(N, \frac{K+1}{K}\right)
$$


from [30, Corollary 1] where $\beta(s, t)$ is the beta function defined in (2). The unconditional expectation then follows as

$$
\begin{aligned}
\mathrm{E}\left[\nu_{\mathrm{opt}}^{(k)}\right] & \leq \mathrm{E}\left[\mathrm{E}\left[\nu_{\mathrm{opt}}^{(k)} \mid \boldsymbol{g}_{k}\right]\right] \\
& =\mathrm{E}\left[\frac{\left\|\boldsymbol{h}_{k}\right\|^{2}}{\left\|\boldsymbol{g}_{k}\right\|^{2}}\right] N \beta\left(N, \frac{K+1}{K}\right) \\
& =\mathrm{E}\left[\frac{\boldsymbol{g}_{k}^{H} \boldsymbol{A} \boldsymbol{A}^{H} \boldsymbol{g}_{k}}{\boldsymbol{g}_{k}^{H} \boldsymbol{g}_{k}}\right] N \beta\left(N, \frac{K+1}{K}\right)
\end{aligned}
$$

To compute $\mathrm{E}\left[\frac{\boldsymbol{g}_{k}^{H} \boldsymbol{A} \boldsymbol{A}^{H} \boldsymbol{g}_{k}}{\boldsymbol{g}_{k}^{H} \boldsymbol{g}_{k}}\right]$, recall that $\boldsymbol{A}^{H} \boldsymbol{A}=\boldsymbol{I} \in$ $\mathbb{R}^{(K+1) \times(K+1)}$. Hence, we can write $\boldsymbol{A} \boldsymbol{A}^{H}=\boldsymbol{T} \boldsymbol{D} \boldsymbol{T}^{H}$ where $D \in \mathbb{R}^{M \times M}$ is a diagonal matrix with $K+1$ elements equal to one and remaining elements equal to zero and $T \in \mathbb{C}^{M \times M}$ is a unitary matrix of orthonormal eigenvectors of $\boldsymbol{A} \boldsymbol{A}^{H}$. Hence, since $\boldsymbol{T} \boldsymbol{T}^{H}=\boldsymbol{I}$, we can write

$$
\begin{aligned}
\mathrm{E}\left[\frac{\boldsymbol{g}_{k}^{H} \boldsymbol{A} \boldsymbol{A}^{H} \boldsymbol{g}_{k}}{\boldsymbol{g}_{k}^{H} \boldsymbol{g}_{k}}\right] & =\mathrm{E}\left[\frac{\boldsymbol{g}_{k}^{H} \boldsymbol{T} \boldsymbol{D} \boldsymbol{T}^{H} \boldsymbol{g}_{k}}{\boldsymbol{g}_{k}^{H} \boldsymbol{T} \boldsymbol{T}^{H} \boldsymbol{g}_{k}}\right] \\
& =\mathrm{E}\left[\frac{\boldsymbol{x}_{k}^{H} \boldsymbol{D} \boldsymbol{x}_{k}}{\boldsymbol{x}_{k} \boldsymbol{x}_{k}}\right] \\
& =\frac{K+1}{M}
\end{aligned}
$$

where we have used the fact that $\boldsymbol{x}_{k}=\boldsymbol{T}^{H} \boldsymbol{g}_{k}$ is isotropic since $\boldsymbol{g}_{k}$ is isotropic and $\boldsymbol{T}$ is unitary. Finally, we use the result

$$
N \beta\left(N, \frac{K+1}{K}\right) \leq N^{-1 / K}
$$

from [17, Appendix II] and the fact that $K+1 \leq M$ to write

$$
\mathrm{E}\left[\nu_{\mathrm{opt}}^{(k)}\right] \leq \frac{K+1}{M} N \beta\left(N, \frac{K+1}{K}\right) \leq N^{-1 / K}
$$

which is the desired result.

The result in Theorem 1 implicitly highlights the benefit of reduced subspace quantization. Note that the scaling is of the form $N^{-1 / K}=2^{-B / K}$, which means the number of bits per dimension is in the exponent. If a standard RVQ quantizer, which uses $\frac{B}{M-1}$ bits per dimension, is employed in a system without receiver coordination, the scaling is of the form $N^{-1 /(M-1)}$. The improvement in the scaling is due to the fact that a reduced subspace allows us to quantize using $B / K$ bits per dimension.

The following Corollary establishes an exact result for the performance of nullforming in the special case of a single primary receiver $(K=1)$ when the primary receiver uses the feedback protocol in Section II-A.

Corollary 1 . In a system with $K=1$ primary receiver and $M>K$ transmit antennas, the expected value of the minimum received power over an $N$-vector $R V Q$ codebook is

$$
\mathrm{E}\left[\nu_{\mathrm{opt}}\right]=\frac{2}{M} N \beta(N, 2)
$$

when the primary receiver feeds back the index of its best beamforming vector.

Proof: Referring to the exact expression for $\nu_{\mathrm{opt}}^{(k)}$ in (10) and since $K=1$, we know $\boldsymbol{n}_{k} \in \mathbb{C}^{2}$. Moreover, since both $\boldsymbol{n}_{k}$ and $\boldsymbol{v}_{\mathrm{opt}}$ are orthogonal to $\boldsymbol{v}_{k, i_{\mathrm{opt}}^{(k)}}$, they must be collinear in a two-dimensional vector space. Hence $\left|\boldsymbol{v}_{\text {opt }}^{H} \boldsymbol{n}_{k}\right|=1$ and

$$
\nu_{\mathrm{opt}}^{(k)}=\frac{\left\|\boldsymbol{h}_{k}\right\|^{2}}{\left\|\boldsymbol{g}_{k}\right\|^{2}} \cdot\left(1-\nu_{\max }^{(k)}\right) .
$$

The desired result then follows directly from (11) and the subsequent analysis in the proof of Theorem 1 .

Note that Theorem 1, while applicable to general $K<M$, only provides an upper bound on the expected received power. Corollary 1 , while restricted to the case when $K=1$, establishes an exact result for the expected receive power when nullforming to a single primary receiver. Also note that an upper bound for the single primary receiver case follows from the fact that $\beta(N, 2) \leq N^{-2}$ as

$$
\mathrm{E}\left[\nu_{\mathrm{opt}}^{(k)}\right] \leq \frac{2}{M N}
$$

which becomes tight as $N \rightarrow \infty$.

In addition, the results in Theorem 1 and Corollary 1 can easily be interpreted in terms of non-normalized SNR. Using (4) and (6), the SNR of the $k$ th user is given by

$$
\mathrm{SNR}_{k}=\frac{\left|\boldsymbol{w}_{\mathrm{opt}}^{H} \boldsymbol{g}_{k}\right|^{2} P}{\sigma^{2}}=\nu_{\mathrm{opt}}^{(k)} \frac{\left\|\boldsymbol{g}_{k}\right\|^{2} P}{\sigma^{2}},
$$

and the average SNR across all users is

$$
\overline{\mathrm{SNR}}=\frac{P}{K \sigma^{2}} \sum_{k=1}^{K} \nu_{\mathrm{opt}}^{(k)}\left\|\boldsymbol{g}_{k}\right\|^{2} .
$$

The average SNR can be easily bounded as

$$
\begin{aligned}
\overline{\mathrm{SNR}} & \leq \frac{P \max _{k}\left\|\boldsymbol{g}_{k}\right\|^{2}}{K \sigma^{2}} \sum_{k=1}^{K} \nu_{\mathrm{opt}}^{(k)} \\
& =\frac{P \max _{k}\left\|\boldsymbol{g}_{k}\right\|^{2}}{\sigma^{2}} \nu_{\mathrm{opt}} \\
& \leq \frac{P}{\sigma^{2}}\left(\sum_{\ell=1}^{K}\left\|\boldsymbol{g}_{\ell}\right\|^{2}\right) \nu_{\mathrm{opt}} .
\end{aligned}
$$

We can further average over the channel distribution to yield

$$
\mathrm{E}[\overline{\mathrm{SNR}}] \leq \frac{P M K}{\sigma^{2}} \mathrm{E}\left[\nu_{\mathrm{opt}}\right]
$$

\section{B. Nullforming with Primary Receiver Coordination}

In the case with receiver coordination, the primary receivers exchange channel estimates and find the codebook vector that minimizes the normalized received power averaged over the primary receivers. The average normalized received power when the transmitter uses precoding vector $\boldsymbol{w}_{i}$ can be written as

$$
\nu_{i}=\frac{1}{K} \sum_{k=1}^{K}\left|\boldsymbol{w}_{i}^{H} \tilde{\boldsymbol{g}}_{k}\right|^{2} .
$$

The minimum average normalized received power over the common $N$-vector RVQ codebook is then

$$
\nu_{\min }=\min _{i \in\{1, \ldots, N\}} \nu_{i}
$$

The following Theorem establishes an upper bound on the expected value of $\nu_{\min }$. 
Theorem 2. In a MIMO system with $K \geq 1$ primary receivers with a common $N$-vector $R V Q$ codebook and $M>K$ transmit antennas, the expected value of the minimum average normalized received power with receiver coordination is upper bounded as

$$
\mathrm{E}\left[\nu_{\min }\right] \leq N \beta\left(N, \frac{K+1}{K}\right) \leq N^{-1 / K} .
$$

Proof: Since the normalized channel matrix $\tilde{\boldsymbol{G}}$ defined in (3) spans at most a $K$-dimensional subspace of $\mathbb{C}^{M}$, we can apply a unitary matrix $\boldsymbol{U} \in \mathbb{C}^{M \times M}$ to the channels and let $\tilde{\boldsymbol{F}}=\boldsymbol{U} \tilde{\boldsymbol{G}}$ correspond to a rotated version of the normalized channel vectors such that $(M-K)$ rows of $\tilde{\boldsymbol{F}}$ are equal to zero. Without loss of generality, we will assume $\boldsymbol{U}$ is chosen such that the final $(M-K)$ rows of $\tilde{\boldsymbol{F}}$ are equal to zero. Denoting

$$
\tilde{\boldsymbol{F}}=\left[\tilde{\boldsymbol{f}}_{1}, \ldots, \tilde{\boldsymbol{f}}_{K}\right] \in \mathbb{C}^{M \times K}
$$

and

$$
\tilde{\boldsymbol{f}}_{k}=[\tilde{f}_{1, k}, \ldots, \tilde{f}_{K, k}, \underbrace{0, \ldots, 0}_{M-K \text { zeros }}]^{\top}
$$

we can then write

$$
\begin{aligned}
\nu_{i} & =\frac{1}{K} \sum_{k=1}^{K}\left|\boldsymbol{w}_{i}^{H} \boldsymbol{U}^{H} \tilde{\boldsymbol{f}}_{k}\right|^{2} \\
& =\frac{1}{K} \sum_{k=1}^{K}\left|\left(\boldsymbol{w}_{i}^{H} \boldsymbol{U}^{H}\right) \sum_{\ell=1}^{K} \tilde{f}_{\ell, k} \boldsymbol{e}_{\ell}\right|^{2}
\end{aligned}
$$

where $\boldsymbol{e}_{\ell}$ is the $\ell^{\text {th }}$ standard basis vector in $\mathbb{R}^{M}$. Letting $\boldsymbol{c}_{i}=\boldsymbol{U} \boldsymbol{w}_{i}=\left[c_{i, 1}, \ldots, c_{i, M}\right]^{\top}$ and noting that $\boldsymbol{c}_{i}$ is also an isotropically distributed unit vector in $\mathbb{C}^{M}$, we can further write

$$
\begin{aligned}
\nu_{i} & =\frac{1}{K} \sum_{k=1}^{K}\left|\boldsymbol{c}_{i}^{H} \sum_{\ell=1}^{K} \tilde{f}_{\ell, k} \boldsymbol{e}_{\ell}\right|^{2} \\
& \leq \frac{1}{K} \sum_{k=1}^{K} \sum_{\ell=1}^{K}\left|c_{i, \ell}\right|^{2}\left|\tilde{f}_{\ell, k}\right|^{2} \\
& \leq \sum_{\ell=1}^{K}\left|c_{i, \ell}\right|^{2} \\
& =\eta_{i}
\end{aligned}
$$

for $i=1, \ldots, N$. Note that the first inequality results from the Cauchy-Shwarz inequality and the second inequality results from the fact that $\left|\tilde{f}_{\ell, k}\right|^{2} \leq 1$ for all $\ell$ and $k$. Also note that $\eta_{i}$ is a random variable that upper bounds $\nu_{i}$ for all channel and precoding vector codebook realizations.

The cumulative distribution function (CDF), denoted by $F_{\eta_{i}}(\cdot, L)$, and probability density function (PDF), denoted by $f_{\eta_{i}}(\cdot, L)$, of $\eta_{i}$ are derived in (21) and (22), respectively, in Appendix A with $L=M-K$. While it is difficult to use these results directly to compute the $\mathrm{CDF}$ and PDF of the minimum

$$
\eta_{\min }=\min _{i \in\{1, \ldots, N\}} \eta_{i}
$$

for general $L$, Lemma 1 in Appendix B establishes that a random variable drawn from $F_{\eta_{i}}(x, L)$ first-order stochastically dominates a random variable drawn from $F_{\eta_{i}}(x, L+1)$. A consequence of this result is that a random variable drawn from $F_{\eta_{i}}(x, L)$ will have larger expected value than a random variable drawn from $F_{\eta_{i}}(x, L+1)$ [33]. Hence, we can upper bound the expected value of $\eta_{i}$ by assuming $L$ takes on its smallest possible value, i.e., $L=1$. Under this assumption, the CDF and PDF of $\eta_{i}$ in (21) and (22) simplify to

$$
\begin{aligned}
& F_{\eta_{i}}(x, 1)=1-(1-x) \sum_{k=0}^{K-1} x^{k}=x^{K} \\
& f_{\eta_{i}}(x, 1)=K x^{K-1} .
\end{aligned}
$$

Since $\left\{\eta_{1}, \ldots, \eta_{N}\right\}$ are independent and identically distributed (i.i.d.), the PDF of $\eta_{\min }$ can then be expressed as

$$
\begin{aligned}
f_{\eta_{\text {min }}}(x, 1) & =N f_{\eta_{i}}(x, 1)\left(1-F_{\eta_{i}}(x, 1)\right)^{N-1} \\
& =N\left(K x^{K-1}\right)\left(1-x^{K}\right)^{N-1}
\end{aligned}
$$

when $L=1$. The expected value of $\eta_{\min }$ when $L=1$ then follows as

$$
\begin{aligned}
\mathrm{E}\left[\eta_{\min }\right] & =N K \int_{0}^{1} x^{K}\left(1-x^{K}\right)^{N-1} d x \\
& =N \beta\left(N, \frac{K}{K+1}\right) \\
& \leq N^{-1 / K}
\end{aligned}
$$

which shows the desired result.

While Theorem 2 provides a general bound for the performance of nullforming with receiver coordination, the following Corollary establishes an exact result for the performance of nullforming in the special case of a single primary receiver $(K=1)$. In this case, while no receiver coordination occurs, the primary receiver uses the coordinated feedback protocol from Section II-B where the feedback corresponds to the index of the primary receiver's best nullforming vector.

Corollary 2. In a system with $K=1$ primary receiver and $M>K$ transmit antennas, the expected value of the minimum received power over an $N$-vector $R V Q$ codebook is

$$
\mathrm{E}\left[\nu_{\min }\right]=\frac{1}{N(M-1)+1}
$$

when the primary receiver feeds back the index of its best nullforming vector.

Proof: In the case with $K=1$ primary receivers, we have the normalized received power with precoding vector $i$ as

$$
\nu_{i}=\left|\boldsymbol{w}_{i}^{H} \tilde{\boldsymbol{g}}\right|^{2}
$$

where we have omitted the $k$ index since there is only a single primary receiver. Given $L=M-1$ and an isotropically distributed precoding vector $\boldsymbol{w}_{i}$, the CDF of $\nu_{i}$ can be written as [11], [30]

$$
F_{\nu_{i}}(x, L)= \begin{cases}0 & x<0 \\ 1-(1-x)^{L} & 0 \leq x \leq 1 \\ 1 & x>1 .\end{cases}
$$

Since the precoding vectors $\boldsymbol{w}_{i}$ are i.i.d., we can write

$$
\operatorname{Prob}\left(\nu_{\min }>x\right)=\left(1-F_{\nu_{i}}(x, L)\right)^{N}=\left((1-x)^{L}\right)^{N}
$$


where $\nu_{\min }$ is defined in (16). Hence, the CDF of $\nu_{\min }$ with an $N$-vector codebook can be written as

$$
F_{\nu_{\min }}(x, L)= \begin{cases}0 & x<0 \\ 1-(1-x)^{N L} & 0 \leq x \leq 1 \\ 1 & x>1\end{cases}
$$

and the PDF of $\nu_{\min }$ follows as

$$
f_{\nu_{\text {min }}}(x, L)= \begin{cases}N L(1-x)^{N L-1} & 0 \leq x \leq 1 \\ 0 & \text { otherwise }\end{cases}
$$

The expected value of the received power can then be computed as

$$
\begin{aligned}
\mathrm{E}\left[\nu_{\text {min }}\right] & =N L \int_{0}^{1} x(1-x)^{N L-1} d x \\
& =\frac{1}{N L+1} \\
& =\frac{1}{N(M-1)+1}
\end{aligned}
$$

which shows the desired result.

Note that (19) can be written as

$$
\mathrm{E}\left[\nu_{\min }\right] \leq \frac{M /(M-1)}{M N}
$$

and this upper bound is tight as $N \rightarrow \infty$. The upper bound in (20) is equal to the upper bound in (13) when $M=2$ and strictly less than the upper bound in (13) when $M>2$. Since the results in (13) and (20) are tight for large $N$, these results show that a $K=1$ system with best nullforming vector feedback as described in Section II-B outperforms the same system with best beamforming vector feedback as described in Section II-A by a factor of $2(M-1) / M$ when the RVQ codebook is large. As the number of antennas also becomes large, i.e. $M \gg 1$, this performance gain becomes a factor of two, or $3 \mathrm{~dB}$.

As in the case without primary receiver coordination, the results in Theorem 2 and Corollary 2 can also be reformulated in terms of non-normalized SNR. The SNR averaged across users and channel realizations is bounded as

$$
\mathrm{E}[\overline{\mathrm{SNR}}] \leq \frac{K M P}{\sigma^{2}} \mathrm{E}\left[\nu_{\min }\right]
$$

\section{Numerical Results}

This section provides numerical results that confirm the analysis in Section III and compares the performance of RVQ nullforming with and without primary receiver coordination. Monte-Carlo simulation results with 1000 iterations are plotted against the analytical results from Section III. In each iteration of the Monte-Carlo simulation, the channel vectors $\boldsymbol{g}_{k} \in \mathbb{C}^{M}$ are drawn i.i.d. from $\mathcal{C N}(0,2 \boldsymbol{I})$, with unit variance independent real and imaginary components, and the precoding vector codebook is also randomly generated, independently of the channel. Both the channel vectors and the precoding vectors are normalized.

Fig. 2 shows the mean normalized received power, averaged over the channel and codebook realizations, for a $K=1$ primary receiver system, $M \in\{4,16,64\}$ transmit antennas, and $N=2^{B}$ precoding vectors per codebook with

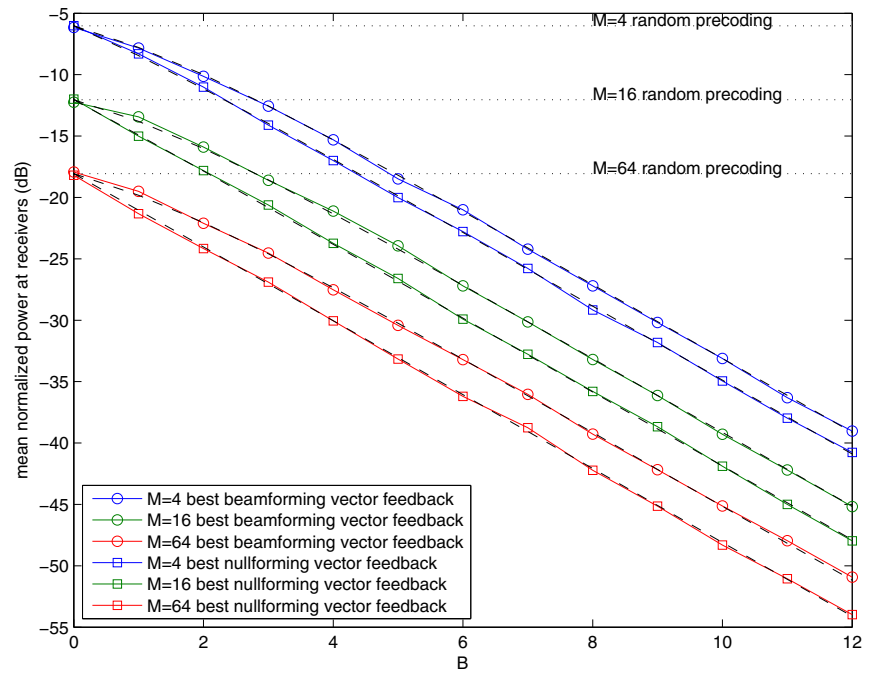

Fig. 2. Mean normalized received power $\mathrm{E}\left[\nu_{\min }\right]$ and $\mathrm{E}\left[\nu_{\mathrm{opt}}\right]$ for a $K=1$ primary receiver system as a function of the codebook size $2^{B}$ and the number of transmit antennas $M$. Solid lines are Monte-Carlo simulation results and dashed lines are analytical results from Section III.

$B \in\{0, \ldots, 12\}$. Since there is only one primary receiver in this case, this is not a comparison of coordinated receivers versus uncoordinated receivers, but rather a comparison of a single primary receiver system with best beamforming vector feedback versus the same system with best nullforming vector feedback. We see that there is close agreement between the analytical results from Corollaries 1 and 2 (dashed black lines) and the Monte-Carlo simulations. Also note the gaps for large $N=2^{B}$ are approximately $1.75 \mathrm{~dB}, 2.75 \mathrm{~dB}$, and $2.95 \mathrm{~dB}$, respectively, for $M=4,16,64$ transmit antennas. These gaps closely agree with the large- $N$ analysis in (13) and (20) showing the performance gain obtained by feeding back best nullforming vectors rather than best beamforming vectors is $2(M-1) / M$.

Fig. 3 shows the mean normalized received power, averaged over the channel and codebook realizations, for a $K=3$ primary receiver system, $M \in\{4,8\}$ transmit antennas, and $N=2^{B}$ precoding vectors per codebook with $B \in\{0, \ldots, 12\}$. We see that the inner bounds in (8) and (17) developed in Theorems 1 and 2, respectively, are both somewhat loose but provide the correct scaling. In fact, the inner bound (8) for $M=4$ and $K=3$ coincides with the inner bound (17) as shown in Fig. 3. The outer bound $N^{-1 / K}$, common to Theorems 1 and 2, is even looser but also provides the correct scaling. Receiver coordination also tends to achieve better nullforming performance, especially as the number of antennas increases as was seen in the $K=1$ case. In this example, when $M=4$ and $N \gg 1$, the performance gap is slightly less than $1 \mathrm{~dB}$, which can be overcome by increasing the codebook size by a factor of 4 without receiver coordination. When $M=8$ and $N \gg 1$, the performance gap is more than $2.5 \mathrm{~dB}$, or approximately a factor of 8 in codebook size.

Fig. 4 shows the mean normalized received power, averaged over the channel and codebook realizations, for a $M=8$ transmit antenna system, $K \in\{1,3,7\}$ primary 


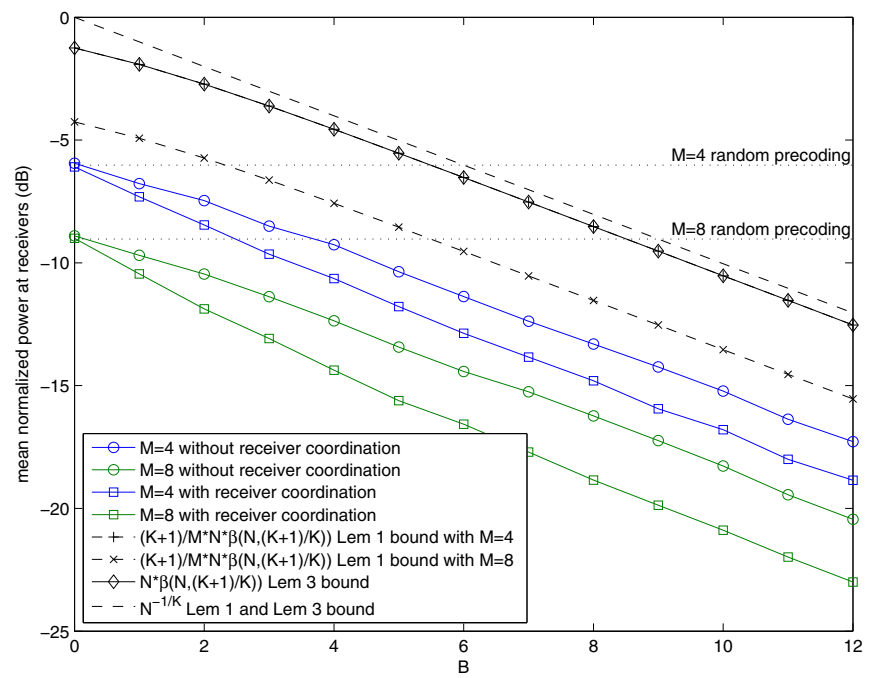

Fig. 3. Mean normalized received power $\mathrm{E}\left[\nu_{\min }\right]$ and $\mathrm{E}\left[\nu_{\mathrm{opt}}\right]$ for a $K=3$ primary receiver system with and without receiver coordination, respectively, as a function of the codebook size $2^{B}$ and the number of transmit antennas $M$. Solid lines are Monte-Carlo simulation results and dashed lines are analytical results from Section III.

receivers, and $N=2^{B}$ precoding vectors per codebook with $B \in\{0, \ldots, 12\}$. In this example, the analytical results were scaled by the mean normalized power of an $M=8$ random precoding vector, i.e., $c=\frac{1}{M}$, to show the scaling results more directly. The performance gap in $\mathrm{dB}$ is similar for all of the tested values of $K$ but, due to the $N^{-1 / K}$ scaling, the codebook size penalty for systems without receiver coordination can be quite large. For example, when $K=7$, a system without receiver coordination requires individual codebooks approximately 32 times larger than the codebook used in a system with receiver coordination to achieve the same performance. This penalty is further exacerbated by the fact that $K B$ bits of feedback are required without receiver coordination, whereas only $B$ bits of feedback are required with receiver coordination.

\section{CONCLUSION}

This paper analyzes the performance of MIMO nullforming with RVQ limited feedback in single-stream systems with and without receiver coordination. The results show that the mean received power at the primary receivers has an upper bound that scales as $N^{-1 / K}=2^{-B / K}$ for nullforming systems with or without receiver coordination. Exact analytical results are also derived for the $K=1$ primary receiver case. Numerical results are provided showing that systems with receiver coordination outperform those without receiver coordination by a constant gap for large $N$ in terms of mean received power.

There are a number of research topics that could build upon this work. First, practical channels often exhibit spatial and temporal correlation. Nullforming codebooks could be designed to utilize spatial correlation rotation techniques similarly to [34], [35]. RVQ codebooks can also be enhanced using techniques to leverage temporal correlation [36]-[43]. This could significantly reduce feedback overhead and improve null

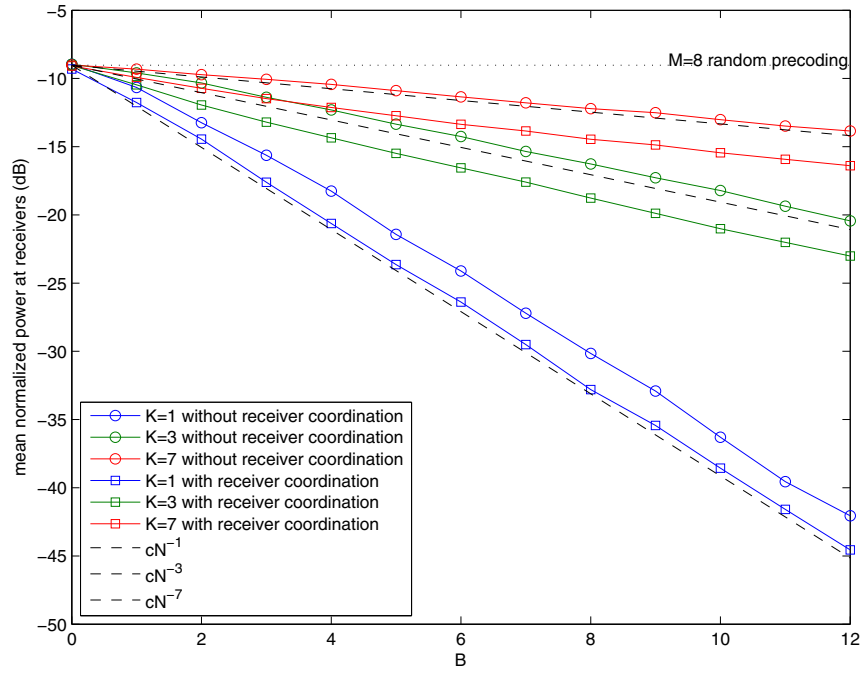

Fig. 4. Mean normalized received power $\mathrm{E}\left[\nu_{\min }\right]$ and $\mathrm{E}\left[\nu_{\mathrm{opt}}\right]$ for a $M=8$ transmit antenna system with and without receiver coordination, respectively, as a function of the codebook size $2^{B}$ and the number of receivers $K$. Solid lines are Monte-Carlo simulation results and dashed lines are analytical results from Section III with scale factor $c=\frac{1}{M}$.

depth. Finally, it is of interest to quantify the performance loss caused by channel estimation error.

\section{APPENDIX A}

\section{Statistical CHARACTERIZATION OF COORDINATED RECEIVER NoRMALIZED RECEIVED POWER BOUND}

From (18), we have defined $\eta_{i}=\sum_{\ell=1}^{K}\left|c_{i, \ell}\right|^{2} \geq \nu_{i}$ as a random variable that upper bounds the normalized received power (averaged over the receivers) over all channel and precoding vector codebook realizations. To compute the CDF of $\eta_{i}$, we can write

$$
\begin{aligned}
F_{\eta_{i}}(x) & =\operatorname{Prob}\left(\sum_{\ell=1}^{K}\left|c_{i, \ell}\right|^{2} \leq x\right) \\
& =\operatorname{Prob}\left(\frac{a}{a+b} \leq x\right) \\
& =\operatorname{Prob}\left(a \leq b\left(\frac{x}{1-x}\right)\right)
\end{aligned}
$$

where $a$ is $\chi$-squared distributed with $2 K$ degrees of freedom, $b$ is $\chi$-squared distributed with $2(M-K)$ degrees of freedom, and $a$ and $b$ are independent. Letting $L=M-K$ and making the dependence of the CDF on $L$ explicit, we can write for $x \in[0,1](21)$ with $F_{\eta_{i}}(x, L)=0$ for $x<0$ and $F_{\eta_{i}}(x, L)=$ 1 for $x>1$. The PDF of $\eta_{i}$ then follows for $x \in[0,1]$ as

$$
\begin{aligned}
f_{\eta_{i}}(x, L)= & L(1-x)^{L-1} \sum_{k=0}^{K-1}\left(\begin{array}{c}
L+k-1 \\
k
\end{array}\right) x^{k} \\
& -(1-x)^{L} \sum_{k=0}^{K-1}\left(\begin{array}{c}
L+k-1 \\
k
\end{array}\right) k x^{k-1}
\end{aligned}
$$

with $f_{\eta_{i}}(x, L)=0$ for $x \notin[0,1]$. 


$$
\begin{aligned}
F_{\eta_{i}}(x, L) & =\int_{0}^{\infty} F_{a}\left(b\left(\frac{x}{1-x}\right)\right) f_{b}(b) d b \\
& =\int_{0}^{\infty}\left(1-e^{-b\left(\frac{x}{1-x}\right)} \sum_{k=0}^{K-1} \frac{1}{k !}\left(b\left(\frac{x}{1-x}\right)\right)^{k}\right) f_{b}(b) d b \\
& =1-\int_{0}^{\infty}\left(e^{-b\left(\frac{x}{1-x}\right)} \sum_{k=0}^{K-1} \frac{1}{k !}\left(b\left(\frac{x}{1-x}\right)\right)^{k}\right) \frac{b^{L-1} e^{-b}}{\Gamma(L)} d b \\
& =1-\sum_{k=0}^{K-1} \frac{x^{k}}{k !(1-x)^{k} \Gamma(L)} \int_{0}^{\infty} e^{-\frac{b}{1-x}} b^{L+k-1} d b \\
& =1-\sum_{k=0}^{K-1} \frac{x^{k}}{k !(1-x)^{k} \Gamma(L)}(1-x)^{L+k} \Gamma(L+k) \\
& =1-(1-x)^{L} \sum_{k=0}^{K-1}\left(\begin{array}{c}
L+k-1 \\
k
\end{array}\right) x^{k}
\end{aligned}
$$

\section{APPENDIX B \\ First ORDER STOCHASTIC DOMINANCE}

The CDF, denoted by $F_{\eta_{i}}(\cdot, L)$, and PDF, denoted by $f_{\eta_{i}}(\cdot, L)$, of the coordinated receiver normalized received power bound $\eta_{i}$ are derived in (21) and (22), respectively, in Appendix A with $L=M-K$. The following Lemma establishes that a random variable drawn from $F_{\eta_{i}}(x, L)$ firstorder stochastically dominates a random variable drawn from $F_{\eta_{i}}(x, L+1)$.

Lemma 1. For fixed $x \in(0,1), K \in \mathbb{N}^{+}, L \in \mathbb{N}^{+}$, a random variable $X$ drawn from $F_{\eta_{i}}(x, L)$, and a random variable $Y$ drawn from $F_{\eta_{i}}(x, L+1)$, we have $X \succ_{\mathrm{FSD}} Y$, i.e. $X$ first order stochastically dominates (FSD) $Y$.

Proof: To show strict FSD, we will show $1-F_{\eta_{i}}(x, L)>$ $1-F_{\eta_{i}}(x, L+1)$, or equivalently

$$
F_{\eta_{i}}(x, L+1)-F_{\eta_{i}}(x, L)>0 .
$$

Defining

$$
\alpha(L, k)=\left(\begin{array}{c}
L+k-1 \\
k
\end{array}\right)
$$

and noting that

$$
\alpha(L+1, k)=\left(\frac{L+k}{L}\right) \alpha(L, k)
$$

from (21) we can write

$$
\begin{aligned}
& \frac{F_{\eta_{i}}(x, L+1)-F_{\eta_{i}}(x, L)}{(1-x)^{L}} \\
& \quad=\sum_{k=0}^{K-1}\left(1-(1-x)\left(\frac{L+k}{L}\right)\right) \alpha(L, k) x^{k} \\
& \quad \stackrel{(a)}{\geq} \sum_{k=0}^{K-1}\left(1-(1-x)\left(\frac{L+k}{L}\right)\right) x^{k} \\
& \quad=x \sum_{k=0}^{K-1} x^{k}-\frac{1}{L}(1-x) \sum_{k=0}^{K-1} k x^{k} \\
& \quad \stackrel{(b)}{\geq} x \sum_{k=0}^{K-1} x^{k}-(1-x) \sum_{k=0}^{K-1} k x^{k} \\
& \quad=K x^{K} \\
& >0
\end{aligned}
$$

where $(a)$ is from the fact that $\alpha(L, k) \geq 1$ and $(b)$ is from the fact that $L \geq 1$ and all of the terms multiplied by $\frac{1}{L}$ in the previous equality are positive. Hence $F_{\eta_{i}}(x, L+1)-$ $F_{\eta_{i}}(x, L)>0$ for all $L \in \mathbb{N}^{+}$and $x \in(0,1)$.

\section{REFERENCES}

[1] F. Rashid-Farrokhi, K. Liu, and L. Tassiulas, "Transmit beamforming and power control for cellular wireless systems," IEEE J. Sel. Areas Commun., vol. 16, no. 8, pp. 1437-1450, 1998.

[2] E. Visotsky and U. Madhow, "Optimum beamforming using transmit antenna arrays," in Proc. 49th IEEE Veh. Technol. Conf., vol. 1, 1999, pp. 851-856.

[3] S. Yi, Y. Pei, and S. Kalyanaraman, "On the capacity improvement of ad hoc wireless networks using directional antennas," in Proc. 2003 ACM International Symp. Mobile Ad Hoc Netw. Comput., ser. MobiHoc '03, pp. 108-116. Available: http://doi.acm.org/10.1145/778415.778429

[4] A. Khisti and G. W. Wornell, "Secure transmission with multiple antennas I: the misome wiretap channel," IEEE Trans. Inf. Theory, vol. 56, no. 7, pp. 3088-3104, 2010.

[5] H. Steyskal, "Synthesis of antenna patterns with prescribed nulls," IEEE Trans. Antennas Propagat., vol. 30, no. 2, pp. 273-279, 1982.

[6] D. Gerlach and A. Paulraj, "Adaptive transmitting antenna arrays with feedback," IEEE Signal Process. Lett., vol. 1, no. 10, pp. 150-152, 1994.

[7] D. J. Love, R. W. Heath Jr., W. Santipach, and M. L. Honig, "What is the value of limited feedback for MIMO channels?" IEEE Commun. Mag., vol. 42, no. 10, pp. 54-59, 2004. 
[8] D. J. Love, R. W. Heath, Jr., V. Lau, D. Gesbert, B. D. Rao, and M. Andrews, "An overview of limited feedback in wireless communication systems," IEEE J. Sel. Areas Commun., vol. 26, no. 8, pp. 1341-1365, Oct. 2008.

[9] T. Yoo, N. Jindal, and A. Goldsmith, "Multi-antenna downlink channels with limited feedback and user selection," IEEE J. Sel. Areas Commun., vol. 25, no. 7, pp. 1478-1491, 2007.

[10] H. Shirani-Mehr and G. Caire, "Channel state feedback schemes for multiuser MIMO-OFDM downlink," IEEE Trans. Commun., vol. 57, no. 9, pp. 2713-2723, 2009.

[11] K. Mukkavilli, A. Sabharwal, E. Erkip, and B. Aazhang, "On beamforming with finite rate feedback in multiple-antenna systems," IEEE Trans. Inf. Theory, vol. 49, no. 10, pp. 2562-2579, 2003.

[12] D. J. Love, R. W. Heath Jr., and T. Strohmer, "Grassmannian beamforming for multiple-input multiple-output wireless systems," IEEE Trans. Inf. Theory, vol. 49, no. 10, pp. 2735-2747, Oct. 2003.

[13] G. Caire, N. Jindal, M. Kobayashi, and N. Ravindran, "Multiuser MIMO achievable rates with downlink training and channel state feedback," IEEE Trans. Inf. Theory, vol. 56, no. 6, pp. 2845-2866, 2010.

[14] J. C. Roh and B. D. Rao, "Efficient feedback methods for MIMO channels based on parameterization," IEEE Trans. Wireless Commun., vol. 6, no. 1, pp. 282-292, Jan. 2007.

[15] - "Design and analysis of MIMO spatial multiplexing systems with quantized feedback," IEEE Trans. Signal Process., vol. 54, no. 8, pp. 2874-2886, Aug. 2006.

[16] V. Raghavan, R. W. Heath, Jr., and A. M. Sayeed, "Systematic codebook designs for quantized beamforming in correlated MIMO channels," IEEE J. Sel. Areas Commun., vol. 25, no. 7, pp. 1298-1310, Sept. 2007.

[17] N. Jindal, "MIMO broadcast channels with finite-rate feedback," IEEE Trans. Inf. Theory, vol. 52, no. 11, pp. 5045-5060, 2006.

[18] P. Ding, D. J. Love, and M. D. Zoltowski, "Multiple antenna broadcast channels with shape feedback and limited feedback," IEEE Trans. Signal Process., vol. 55, no. 7, pp. 3417-3428, July 2007.

[19] D. J. Ryan, I. Clarkson, I. B. Collings, D. Guo, and M. L. Honig, "QAM and PSK codebooks for limited feedback MIMO beamforming," IEEE Trans. Commun., vol. 57, no. 4, pp. 1184-1196, Apr. 2009.

[20] C. K. Au-Yeung, D. J. Love, and S. Sanayei, "Trellis coded line packing: large dimensional beamforming vector quantization and feedback transmission," IEEE Trans. Wireless Commun., vol. 10, no. 6, pp. 18441853, June 2011.

[21] W. Santipach and M. L. Honig, "Asymptotic performance of mimo wireless channels with limited feedback," in Proc. 2003 IEEE Military Commun. Conf., vol. 1, pp. 141-146.

[22] _ "Asymptotic capacity of beamforming with limited feedback," in Proc. 2004 IEEE International Symp. Inf. Theory, p. 290.

[23] W. Dai, Y. Liu, and B. Rider, "Quantization bounds on Grassmann manifolds and applications to MIMO communications," IEEE Trans. Inf. Theory, vol. 54, no. 3, pp. 1108-1123, 2008.

[24] W. Santipach and M. Honig, "Capacity of a multiple-antenna fading channel with a quantized precoding matrix," IEEE Trans. Inf. Theory, vol. 55, no. 3, pp. 1218-1234, 2009.

[25] V. Raghavan and V. V. Veeravalli, "On quantized multi-user beamforming in spatially correlated broadcast channels," in Proc. 2007 IEEE Int. Symp. Inf. Theory, pp. 2041-2045.

[26] D. Ryan, "Performance of RVQ limited feedback beamforming over correlated channels," in Proc. 2010 IEEE Wireless Commun. Netw. Conf.

[27] V. Raghavan, M. L. Honig, and V. V. Veeravalli, "Performance analysis of RVQ codebooks for limited feedback beamforming," in Proc. 2009 IEEE Int. Symp. Inf. Theory, pp. 2437-2441.

[28] W. Santipach and K. Mamat, "Tree-structured random vector quantization for limited-feedback wireless channels," IEEE Trans. Wireless Commun., vol. 10, no. 9, pp. 3012-3019, Sept. 2011.

[29] V. Raghavan and V. V. Veeravalli, "Ensemble properties of RVQ-based limited feedback beamforming codebooks," IEEE Trans. Inf. Theory, 2012, submitted. Available: http://arxiv.org/abs/1207.1524.

[30] C. K. Au-Yeung and D. Love, "On the performance of random vector quantization limited feedback beamforming in a miso system," IEEE Trans. Wireless Commun., vol. 6, no. 2, pp. 458-462, 2007.

[31] M. Abramowitz and I. Stegun, Handbook of Mathematical Functions. Dover, 1972.

[32] G. H. Golub and C. F. V. Loan, Matrix Computations, 3rd ed. The Johns Hopkins University Press, 1996.

[33] J. Hadar and W. R. Russell, "Rules for ordering uncertain prospects," American Economic Rev., vol. 59, no. 1, pp. 25-34, 1969.

[34] D. J. Love and R. W. Heath, Jr., "Limited feedback diversity techniques for correlated channels," IEEE Trans. Veh. Technol., vol. 55, no. 2, pp. 718-722, Mar. 2006.

[35] P. Xia and G. B. Giannakis, "Design and analysis of transmitbeamforming based on limited-rate feedback," IEEE Trans. Signal Process., vol. 54, no. 5, pp. 1853-1863, May 2006.

[36] B. Banister and J. Zeidler, "Feedback assisted transmission subspace tracking for MIMO systems," IEEE J. Sel. Areas Commun., vol. 21, pp. 452-463, May 2003.

[37] J. Yang and D. Williams, "Transmission subspace tracking for MIMO systems with low-rate feedback," IEEE Trans. Commun., vol. 55, no. 8, pp. 1629-1639, Aug. 2007.

[38] R. W. Heath Jr., T. Wu, and A. C. K. Soong, "Progressive refinement of beamforming vectors for high-resolution limited feedback," EURASIP J. Advances Signal Process., vol. 2009, no. 6, Feb. 2009.

[39] K. Huang, R. W. Heath Jr., and J. G. Andrews, "Limited feedback beamforming over temporally-correlated channels," IEEE Trans. Signal Process., vol. 57, no. 5, pp. 1959-1975, May 2009.

[40] D. Sacristan and A. Pascual-Iserte, "Differential feedback of MIMO channel gram matrices based on geodesic curves," IEEE Trans. Wireless Commun., vol. 9, no. 12, pp. 3714-3727, Dec. 2010.

[41] T. Kim, D. J. Love, and B. Clerckx, "MIMO system with limited rate differential feedback in slow varying channel," IEEE Trans. Commun., vol. 59, no. 4, pp. 1175-1180, Apr. 2010.

[42] J. Choi, B. Clerckx, N. Lee, and G. Kim, "A new design of polarcap differential codebook for temporally/spatially correlated MISO channels," IEEE Trans. Wireless Commun., vol. 11, no. 2, pp. 703-711, Feb. 2012.

[43] J. Choi, B. Clerckx, and D. J. Love, "Differential codebook for general rotated dual-polarized MISO channels," in Proc. 2012 IEEE Global Telecomun. Conf.

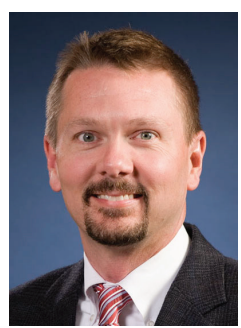

D. Richard Brown III (S'97-M'00-SM'09) received the B.S. and M.S. degrees in Electrical Engineering from The University of Connecticut in 1992 and 1996, respectively, and received the Ph.D. degree in Electrical Engineering from Cornell University in 2000. From 1992-1997, he was with General Electric Electrical Distribution and Control. He joined the faculty at Worcester Polytechnic Institute (WPI) in Worcester, Massachusetts in 2000 and currently is an Associate Professor. He also held an appointment as a Visiting Associate Professor at Princeton University from August 2007 to June 2008. His research interests are currently in coordinated wireless transmission and reception, synchronization, distributed computing, and game-theoretic analysis of communication networks.

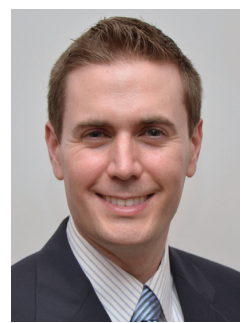

David J. Love (S'98-M'05-SM'09) received the B.S. (with highest honors), M.S.E., and Ph.D. degrees in electrical engineering from the University of Texas at Austin in 2000, 2002, and 2004, respectively. During the summers of 2000 and 2002, he was with Texas Instruments, Dallas, TX. Since August 2004, he has been with the School of Electrical and Computer Engineering, Purdue University, West Lafayette, IN, where he is now a Professor and recognized as a University Faculty Scholar. He has served as an Associate Editor for both the IEEE TRANSACTIONS ON COMMUNICATIONS and the IEEE TRANSACTIONS ON SignAl PROCESSING, and he has also served as a guest editor for special issues of the IEEE JOURNAL ON SELECTED AREAS IN COMMUNICATIONS and the EURASIP Journal on Wireless Communications and Networking. His research interests are in the design and analysis of communication systems and MIMO array processing. He has published over 120 technical papers in these areas and filed more than 20 U.S. patents, 19 of which have issued.

Dr. Love has been inducted into Tau Beta Pi and Eta Kappa Nu. Along with co-authors, he was awarded the 2009 IEEE Transactions on Vehicular Technology Jack Neubauer Memorial Award for the best systems paper published in the IEEE TRANSACTIONS ON VEHICULAR TECHNOLOGY in that year and a 2013 IEEE Global Communications conference best paper award. He was the recipient of the Fall 2010 Purdue HKN Outstanding Teacher Award, the Fall 2013 Purdue ECE Graduate Student Association Outstanding Professor Award, and was an invited participant to the 2011 NAE Frontiers of Engineering Education Symposium. In 2003, Dr. Love was awarded the IEEE Vehicular Technology Society Daniel Noble Fellowship. 